\title{
Elaboración de vídeos educativos sobre operaciones básicas de laboratorio
}

\author{
Cristina García \\ María Pilar Ribate \\ Laura Culleré \\ Henar Fernández \\ Beatriz Giner \\ Universidad San Jorge, España
}

\section{Resumen}

Se presenta una iniciativa en la que alumnos de primer curso del Grado en Farmacia de la Universidad San Jorge crearon vídeos educativos sobre operaciones básicas de laboratorio. Los alumnos de tercer y cuarto curso de la ESO del Centro San Valero utilizan el material para conocer cómo realizar dichas operaciones. Para los alumnos universitarios, se han diseñado una serie de actividades que además de exigir que apliquen lo aprendido, les obliga a identificar y sintetizar puntos críticos de determinadas operaciones para la elaboración de un vídeo. Asimismo, emplean el inglés como lengua de comunicación, demuestran su destreza y trabajan en grupo. El servicio consiste en emplear los vídeos para formar a alumnos de Educación Secundaria antes de introducirlos en un laboratorio ya que, dada la situación de pandemia, no pueden asistir a las prácticas. Los vídeos se emplean como elemento motivador, que les permita afrontar la entrada al laboratorio con una mejor formación.

\section{Palabras clave}

Innovación, vídeos educativos, laboratorio, universidad, educación secundaria.

Fecha de recepción: 28/IX/2020

Fecha de aceptación: 6/XI/2020 


\title{
Creation of educational videos about basic laboratory operations
}

\begin{abstract}
This paper discusses an initiative through which first-year students of the degree in Pharmacy at San Jorge University (USJ) created educational videos about basic operations carried out in the laboratory, aimed at students in Compulsory Secondary Education (ESO) at the San Valero Centre. In addition, the university students used English as their language of communication, demonstrated their skill in handling laboratory material and worked in groups, organising themselves with the aim of completing a common task. $95 \%$ of the participating ESO students rated the activity 8 points or higher out of 10 , while $83 \%$ of the university students considered it suitable for the Degree in Pharmacy, rating it 8 points out of 10 . It is worth highlighting that the students receiving the service pointed out the usefulness of the videos in learning how to carry out certain basic laboratory operations and that the teachers recognised that the videos have been useful and interesting for training the students.
\end{abstract}

\section{Keywords}

Innovation, educational videos, laboratory, university, secondary education. 


\section{Introducción ${ }^{1}$}

La situación pandémica a la que nos hemos enfrentado en el curso 2019-20 ha supuesto un desafío educativo para los docentes teniendo que adaptarnos en un breve espacio de tiempo a la nueva situación sin presencialidad en el aula. La docencia online cuenta tanto con ventajas como inconvenientes, pero especialmente exige una buena planificación de los docentes en cuanto a las actividades a realizar (Pardo, 2014). Una posible herramienta que emplear son los vídeos educativos (Cabero, 1995). La naturaleza y el origen de estos vídeos puede ser muy variado, pero si no se ajustan bien a las necesidades de la materia, al nivel educativo de los alumnos o al contexto del centro pueden resultar ineficaces para el objetivo didáctico que se pretende alcanzar.

Los vídeos educativos también han sido objeto de estudio como buenas herramientas docentes para una mejor comprensión y asimilación de contenidos en materias experimentales o con contenidos prácticos (Cameán et al., 2005; Rodríguez, Uría, y Carreño, 2007).

En esta iniciativa se han pretendido fusionar ambas aproximaciones con un enfoque de aprendizaje-servicio. Concretamente, se encontraría en línea con la nueva modalidad de aprendizajeservicio en escenarios digitales de

\footnotetext{
${ }^{1}$ Las autoras desean agradecer la participación de todos los alumnos y docentes de los dos centros educativos implicados en esta experiencia de aprendizaje-servicio.
}

aprendizaje (Ruiz-Corbella y GarcíaGutiérrez, 2020). Los alumnos universitarios del Grado en Farmacia elaboraron vídeos educativos aplicando los conocimientos adquiridos en la asignatura de Introducción al Trabajo de Laboratorio (ITL). Estos vídeos se emplearon en el centro de Educación Secundaria San Valero para mostrar a sus alumnos cómo desarrollar operaciones básicas de laboratorio en el contexto de asignaturas de Física y Química, en la parte de servicio.

\section{Breve marco teórico}

Actualmente se apuesta por metodologías activas de enseñanzaaprendizaje en las que se otorga al alumno un mayor protagonismo en su proceso de formación. Muchas de ellas incluyen actividades de innovación docente y las experiencias de aprendizaje-servicio forman parte de este grupo. Se trata de una combinación del aprendizaje basado en la experiencia y el servicio a la comunidad (Batlle, 2011). Además, el aprendizaje-servicio como recurso educativo puede mejorar el compromiso cívico y las habilidades intelectuales de los alumnos, ya que nutre su desarrollo social y personal, mejorando sus habilidades orales y escritas.

Por lo tanto, únicamente tiene sentido si se abordan ambas vertientes, identificar un problema social definido, que formará parte del servicio, y concretar los resultados de aprendizaje que se pretenden conseguir en los alumnos.

El recurso seleccionado ha sido la 
elaboración de vídeos por parte de alumnos y la posterior visualización por otro grupo de distintos niveles educativos, en el contexto de diferentes asignaturas de la formación académica que están recibiendo. Se espera que los vídeos tengan una elevada potencialidad expresiva, superior a otros recursos como presentaciones con diapositivas (Bravo Ramos, 1996). Se trata de recursos útiles en el proceso de enseñanza-aprendizaje porque habitualmente captan la atención del público, especialmente si son de corta duración (Pérez, Rodríguez y García, 2014). La capacidad de transmisión de un vídeo es alta ya que combina imágenes, sonidos e incluso se puede añadir texto durante el proceso de edición expresando el contenido de una forma más completa, siendo especialmente importante la estrategia didáctica en la que se pretenda integrar (Cabero, 1995; García Matamoros, 2014).

Sobre la temática que engloba el proyecto (trabajo en laboratorio), existen iniciativas de elaboración de vídeos educativos, aunque ninguna integra específicamente el enfoque mostrado en este proyecto. En otras iniciativas se elaboraron vídeos para tratar el tema de la seguridad en los laboratorios con alumnos del Grado en Farmacia (Cameán et al., 2005).

También se ha publicado una experiencia en la que se introducía el uso de vídeos en la asignatura de Química, los alumnos consultaban los vídeos que los docentes ponían a su disposición para mejorar su formación (Menéndez, Van der Maelen y Pérez, 2005). En Educación Secundaria encontramos experiencias con vídeos en las que los alumnos participaban activamente en la grabación de un experimento, en el área de la Física y Química (Torres, 2017).

\section{Descripción de la experiencia}

Se pretende alcanzar el objetivo de aprendizaje, principalmente en los alumnos de las asignaturas ITL y Química Orgánica (QO) del Grado en Farmacia. La asignatura de ITL tiene como objetivo principal proporcionar al estudiante el conocimiento de las normas de seguridad, del control de calidad del funcionamiento y de los principios básicos de los métodos de análisis de un laboratorio. Pertenece al bloque formativo de Química y por tanto se incide en aquellos aspectos que más interés pueden presentar en esta materia. Los alumnos matriculados en esta asignatura asisten, dentro de las horas presenciales de la asignatura, a 16 horas de formación práctica en el laboratorio. Uno de los resultados de aprendizaje incluye manipular los instrumentos básicos del laboratorio con destreza. Además, el Grado en Farmacia tiene como objetivo la formación de profesionales expertos en el medicamento y en su impacto en la salud. La práctica totalidad de los fármacos están formados por moléculas orgánicas y por ello es necesario que el futuro farmacéutico conozca sus características tanto físicas como químicas. El módulo Química y en concreto, la asignatura QO proporcionan al estudiante los conocimientos necesarios para relacionar la estructura de una molécula orgánica con las reacciones que ésta puede experimentar, así como aplicar la 
información recibida a la síntesis de nuevos compuestos que posean las características deseadas. Los alumnos de esta materia realizan 6 sesiones de laboratorio (24 horas) donde aprenden las técnicas básicas del laboratorio de QO: preparación de reactivos, síntesis, purificación y análisis. Para ello, han de aplicar los conocimientos y destrezas aprendidos previamente en la asignatura de ITL.

Las actividades de enseñanzaaprendizaje diseñadas en ITL una vez recibida la información teórica y práctica fueron:

1) Elaboración de un vídeo educativo por parte de los alumnos mostrando la información más relevante de alguna operación básica de laboratorio. Esta actividad forma parte del programa de integración de lengua inglesa y el vídeo debía elaborarse en inglés, practicando el vocabulario científico aprendido como parte de la asignatura. Los nuevos modelos de aprendizaje están centrados en el alumno. En esta actividad se pretende que aprendan de forma activa, poniendo en práctica lo aprendido con anterioridad. Para ello debían ser capaces de identificar los puntos más importantes de la operación asignada, sintetizarla y mostrarla en un vídeo de un máximo de 2 minutos. Para alcanzar este objetivo, debían elaborar un guion del vídeo, un inventario de material necesario para la grabación y reservar un espacio de laboratorio durante 30 minutos, realizar y grabar la operación. Para la edición posterior del vídeo se recomendó el empleo de herramientas como Movie Maker, FilmmoraGo, WeVideo o iMovie, dependiendo de sus soportes electrónicos de preferencia.

El contenido de los vídeos incluyó las siguientes operaciones:

- preparación de una disolución a partir de un compuesto sólido y un compuesto líquido,

- ensamblaje del equipo de destilación simple,

- pipeteo con diferentes pipetas y micropipetas,

- uso de la bureta y del pH-metro.

La actividad se realizó en grupo, desarrollando también la capacidad de gestionar un grupo de trabajo e integrarse en el mismo. Se potenció igualmente el empleo de las tecnologías de la información y la comunicación (TIC) y la lengua inglesa, ya que forma parte de las actividades CLIL (Content and Language Integrated Learning) de la asignatura. En el curso 2019-20 se contó con 42 alumnos en la asignatura y se elaboraron un total de 13 vídeos (1/2 vídeos por tema propuesto).

2) Presentación en clase de los vídeos elaborados $(n=13)$. Se pretendía que los alumnos mostrasen el trabajo realizado y exponiéndolo al juicio de sus compañeros que, al azar, se elegían para destacar algún aspecto positivo del trabajo, así como propuestas de mejora. De esta forma se potencia el espíritu crítico de los alumnos, y la reflexión sobre el trabajo realizado.

3) Los trabajos se sometieron a evaluación mediante una rúbrica (tabla 1), que los alumnos disponían desde el

García, C., Ribate, M.P., Culleré, L., Fernández, H. y Giner, B. (2020). Elaboración de vídeos educativos sobre operaciones básicas de laboratorio. RIDAS, Revista Iberoamericana de Aprendizaje Servicio, 10, 126-136. DOI10.1344/RIDAS2020.10.11 
inicio. Junto con la calificación obtenida tenían una retroalimentación en relación con la misma.

Tabla 1. Rúbrica de evaluación de los vídeos elaborados por los alumnos del Grado en Farmacia

\begin{tabular}{|c|c|c|c|}
\hline Items & 2 & 1 & 0,5 \\
\hline Planning & $\begin{array}{l}\text { All task have } \\
\text { been } \\
\text { developed } \\
\text { according to } \\
\text { the timetable. }\end{array}$ & $\begin{array}{l}\text { Most tasks } \\
\text { have been } \\
\text { developed } \\
\text { according to } \\
\text { the } \\
\text { timetable. }\end{array}$ & $\begin{array}{l}\text { A few tasks } \\
\text { have been } \\
\text { developed } \\
\text { according } \\
\text { to the } \\
\text { timetable. }\end{array}$ \\
\hline Appearance & $\begin{array}{l}\text { Presents the } \\
\text { information } \\
\text { through a } \\
\text { original way. } \\
\text { Images } \\
\text { enhance the } \\
\text { understanding } \\
\text { of the topic. } \\
\text { Structured }\end{array}$ & $\begin{array}{l}\text { Some } \\
\text { aspects of } \\
\text { the video } \\
\text { are original. } \\
\text { Images are } \\
\text { directly } \\
\text { connected } \\
\text { to the } \\
\text { information. } \\
\text { Structured. }\end{array}$ & $\begin{array}{l}\text { Video is } \\
\text { predictable, } \\
\text { without } \\
\text { original } \\
\text { ideas. } \\
\text { Images } \\
\text { support } \\
\text { vaguely the } \\
\text { main ideas } \\
\text { about the } \\
\text { topic. } \\
\text { Structured } \\
\text { defects. }\end{array}$ \\
\hline Content & $\begin{array}{l}\text { Shows } \\
\text { excellent } \\
\text { application of } \\
\text { vocabulary } \\
\text { related topic. } \\
\text { Includes the } \\
\text { most relevant } \\
\text { information } \\
\text { about the } \\
\text { topic. }\end{array}$ & $\begin{array}{l}\text { Shows good } \\
\text { application } \\
\text { of some } \\
\text { vocabulary } \\
\text { related } \\
\text { topic. Only } \\
\text { Includes } \\
\text { some } \\
\text { relevant } \\
\text { information } \\
\text { about the } \\
\text { topic. }\end{array}$ & $\begin{array}{l}\text { Shows } \\
\text { incorrect } \\
\text { application } \\
\text { of some } \\
\text { terms. } \\
\text { Some } \\
\text { relevant } \\
\text { ideas are } \\
\text { missing. }\end{array}$ \\
\hline $\begin{array}{l}\text { Clarity and } \\
\text { fluency }\end{array}$ & $\begin{array}{l}\text { English } \\
\text { language is } \\
\text { clear. Content } \\
\text { easy to } \\
\text { follow. }\end{array}$ & $\begin{array}{l}\text { English } \\
\text { language is } \\
\text { clear. } \\
\text { Content } \\
\text { easy to } \\
\text { follow. }\end{array}$ & $\begin{array}{l}\text { English } \\
\text { language is } \\
\text { clear. } \\
\text { Content } \\
\text { easy to } \\
\text { follow. }\end{array}$ \\
\hline Extension & $\begin{array}{l}\text { Between 1,5- } \\
2 \text { minutes. }\end{array}$ & $\begin{array}{l}\text { Almost } 1,5- \\
2 \text { minutes. }\end{array}$ & $\begin{array}{l}\text { Different } \\
\text { from 1,5-2 } \\
\text { minutes. }\end{array}$ \\
\hline
\end{tabular}

Fuente: elaboración propia
Además de la evaluación de los vídeos por parte de las docentes de la asignatura ITL, se analizó su calidad y utilidad como información previa para los alumnos antes de comenzar prácticas de otras asignaturas, conjuntamente con otras docentes del Grado.

4) Generalmente, los docentes de otras asignaturas perciben que es necesario recordar a los alumnos los conocimientos de las operaciones más básicas de laboratorio para trabajar correctamente en él. Para comprobar la utilidad de estos vídeos, los alumnos de la asignatura QO contaron con una actividad de flipped-learning en la que visualizaron los vídeos seleccionados en la fase anterior, como un pase previo al comienzo de las prácticas de la asignatura. Además, cumplimentaron un cuestionario online obligatorio para comprobar que habían hecho un aprovechamiento adecuado de los vídeos. Estos estaban encaminados a reforzar los conceptos vistos en los vídeos, detectar errores (si los hubiera) y extrapolar la información recibida en el momento de realizar las prácticas de QO. Posteriormente los alumnos participantes evaluaron la actividad.

Tras la finalización de las actividades académicas, los docentes de la US] seleccionaron los mejores vídeos $(n=7)$ para emplearlos en la parte de servicio del proyecto. Las autorizaciones de los alumnos universitarios para compartir el material elaborado con su propiedad intelectual en el contexto educativo se redactaron en colaboración con el Servicio Jurídico de la USJ. También se revisó la limitación del uso de los mismos, contando igualmente con el 
compromiso firmado por parte de los docentes de Educación Secundaria para emplearlos únicamente con la finalidad educativa descrita. Se contó con la autorización de los alumnos de 3 vídeos sobre: preparación de una disolución a partir de un compuesto sólido, empleo de diferentes usos de pipetas y montaje de un equipo de destilación simple.

Las calificaciones obtenidas en el trabajo en grupo de elaboración de los vídeos $(n=13)$ tuvieron una media de $7,8 \pm 2,3$. Cualitativamente, las docentes de la asignatura apreciaron un gran interés de la mayoría de los alumnos por realizar el trabajo de forma adecuada. Demostraron una buena capacidad de organización al entregar a tiempo su planificación a las responsables de laboratorio, anticipando los materiales necesarios para la elaboración del vídeo y sin excederse del tiempo de reserva del laboratorio estimado como oportuno. La herramienta de edición de vídeo más empleada fue iMovie $(n=4)$, seguida por Movie Maker y FilmmoraGo $(n=2$ en ambas). Una vez terminada la actividad, se preguntó acerca del interés de los vídeos como método de recopilación de información para QO, utilidad para la preparación de prácticas, calidad de los vídeos y adecuación de la actividad en general para su formación como Farmacéuticos. Asimismo, se plantearon diversas preguntas de respuestas abierta con el fin de recabar información y mejorar la actividad en futuras ediciones. Los resultados indican que el $100 \%$ de los alumnos encuestados $(n=18)$ consideró que la actividad era muy relevante para el grado, puntuándola con 6 puntos o más. La utilidad de la actividad para preparar las prácticas se valoró en 8,3 $\pm 1,3$. El uso de vídeos como método de recopilación de información se valoró en $8,4 \pm 0,8$. La calidad de los vídeos obtuvo la puntuación más baja $(7,5 \pm$ $0,8)$. Las preguntas de respuesta abierta recogían que la mayor dificultad se había encontrado en el proceso de edición, la utilización del idioma inglés y el tiempo asignado para la grabación en el laboratorio que consideraron escaso (30 minutos por grupo). En general, la mayoría de los alumnos opinó que la actividad debería repetirse en ediciones posteriores al mejorar la preparación de las prácticas de la asignatura QO ayudándoles a afianzar conceptos y a trabajar en equipo.

Existía interés por parte del Centro San Valero de disponer de vídeos educativos relacionados con el área de Química, en particular para suplir las prácticas de laboratorio sin posibilidad de realizarse de forma presencial. Con el objetivo de promover el interés y motivación de su alumnado en la participación activa durante las prácticas de laboratorio, cuando finalmente se puedan desarrollar. Se esperaba que la visualización de las distintas operaciones de forma anticipada a la realización de la práctica in situ pudiera ayudar a comprender mejor determinados conceptos que se trabajan durante prácticas de la asignatura Física y Química, cuando sea posible. De esa forma, el alumno conoce exactamente los pasos que se van a dar y el objetivo al que se quiere llegar, generando un esquema mental previo que le podría proporcionar confianza y claridad mejorando así el 
posterior aprendizaje.

Durante la última semana lectiva de abril del 2020, los diferentes grupos de alumnos de $3^{\circ}$ y $4^{\circ}$ de la ESO del Centro San Valero, visualizaron los 3 vídeos elaborados por los alumnos universitarios durante sus clases online de la asignatura Física y Química a través de la plataforma Google Meet.

Tanto los docentes como los alumnos cumplimentaron unas encuestas en las que se les preguntó acerca de los siguientes aspectos:

1) Puntúa en una escala de 0 (nada) a 10 (mucho) los siguientes aspectos:

1.a) Utilidad de los vídeos para aprender cómo se realizan operaciones básicas de laboratorio.

1.b) Calidad de los vídeos.

1.c) Interés de los vídeos.

2) ¿Qué es lo que más te ha gustado aprender con estos vídeos?

3) ¿Qué otros temas sobre el laboratorio te gustarían aprender con vídeos educativos cortos de este tipo?

4) ¿Qué mejorarías si se volviera a repetir una experiencia de este tipo?

5) ¿Qué es lo que más difícil te ha resultado a la hora de ver y comprender el video (lenguaje, material, procedimiento, ...)?

Los resultados numéricos obtenidos acerca de la utilidad, calidad e interés de los vídeos tras la realización de las encuestas se muestran en la tabla 2 . Se recopilaron un total de 85 encuestas, 6 cumplimentadas por docentes del área de física y química de $3^{\circ}$ y $4^{\circ}$ de ESO y 79 por alumnos de ambos cursos.

Tabla 2. Resultados encuestas de evaluación de proyecto de vídeo educativo ( $n=$ número; $\bar{X}=$ media aritmética; s.d. = desviación estándar) de docentes y alumnos del centro de Educación Secundaria

\begin{tabular}{|c|c|c|c|c|c|c|c|}
\cline { 2 - 7 } \multicolumn{1}{c|}{} & \multicolumn{2}{c|}{ UTILIDAD } & \multicolumn{2}{c|}{ CALIDAD } & \multicolumn{2}{c|}{ INTERÉS } \\
\cline { 2 - 7 } \multicolumn{1}{c|}{} & $\mathrm{n}$ & $\overline{\mathrm{X}}$ & s.d. & $\overline{\mathrm{X}}$ & s.d. & $\overline{\mathrm{X}}$ & s.d. \\
\hline $\begin{array}{c}\text { Docentes } \\
\mathbf{( 3} \text { y 40 } \\
\text { ESO) }\end{array}$ & 6 & 8,5 & 1 & 7,3 & 1,6 & $\mathbf{8 , 6}$ & 1,1 \\
\hline $\begin{array}{c}\text { Alumnos } \\
\mathbf{3 0}^{\circ} \text { ESO }\end{array}$ & 46 & 8,3 & 1,4 & 7,1 & 1,5 & 7,9 & 1,2 \\
\hline $\begin{array}{c}\text { Alumnos } \\
\text { 40 ESO }\end{array}$ & 33 & 8,1 & 1,2 & 7,2 & 1,1 & 7,9 & 1 \\
\hline $\begin{array}{c}\text { Total } \\
\text { alumnos } \\
\text { 30 y 40 } \\
\text { ESO }\end{array}$ & 79 & $\mathbf{8 , 2}$ & 1,3 & 7,1 & 1,3 & 7,9 & 1,1 \\
\hline
\end{tabular}

Fuente: elaboración propia

De forma general se puede observar que, en los tres aspectos evaluados, las evaluaciones de los docentes presentan una media más elevada que las de los alumnos. El aspecto mejor valorado por los docentes fue el interés de los vídeos mientras que en los alumnos la utilidad de estos. En ambos casos la media aritmética más baja vuelve a ser de calidad de los vídeos. 
Resulta interesante comprobar que entre los grupos de $3^{\circ}$ y $4^{\circ}$ no hay diferencias significativas entre las medias ( $t$-student) para ninguno de los aspectos evaluados con relación a los vídeos $(p>0,05)$. Aunque pertenecen a niveles educativos distintos, podemos considerarlos como un grupo único en cuanto a la evaluación del impacto del proyecto.

En los comentarios de las preguntas abiertas, a pesar de contar con opiniones individuales únicas y dispares, especialmente en los temas que más les han gustado, existen ciertos aspectos que se repiten y que merece la pena resaltar. En las evaluaciones de los docentes, hay varias observaciones acerca de que la calidad del vídeo se podría mejorar, así como la velocidad de exposición. Este aspecto coincide con los resultados de la evaluación cuantitativa. Además, como propuestas, sugieren que, al ser en inglés, se añadan subtítulos para un mejor seguimiento del contenido y se estructure la información con una parte introductoria. Aunque sólo un docente lo ha planteado, hay dos alumnos que coinciden en que como posibles temas adicionales se podría contar con un vídeo acerca de las medidas de seguridad que se deben seguir en el trabajo en laboratorio. Por último, uno de los docentes reconoce que "el aprendizaje visual es más sencillo que sólo el teórico sin imagen". Siendo ese uno de los objetivos de la iniciativa, acercar contenido de una forma más accesible y clarificadora a los alumnos. Varios alumnos $(n=10)$ en cambio también lo han destacado como una forma de aprender más sencilla, amena y/o interesante. Esta información concuerda con las ventajas que ya se han destacado en trabajos previos (García Matamoros, 2014). Las propuestas de vídeos adicionales se concentran mayoritariamente en reacciones químicas y en algunos casos en particular añaden que sean visuales, llamativas o que puedan replicar en sus domicilios o en sus clases prácticas en el laboratorio de su centro. Un elevado número de alumnos proponen incluir vídeos sobre temas más relacionados con la biología, e incluso un alumno muestra interés por el tema de actualidad sobre la elaboración de vacunas frente a agentes virales. El uso de subtítulos o incluso la elaboración de los vídeos en castellano es uno de los aspectos a mejorar. Ya que el $61 \%$ de los alumnos señalan el lenguaje como la mayor dificultad para seguir los mismos. También señalan la posible mejora de la calidad del vídeo y/o audio. La motivación de los alumnos se pudo constatar en muchos comentarios generales como "El laboratorio es un tema que me interesa mucho ya que me gustan las ciencias, por eso me ha gustado mucho el vídeo. Lo que más me ha gustado es que expliquen para que sirve cada material y como se mide en ellos antes de utilizarlos directamente".

\section{Conclusiones}

Los vídeos, como elementos curriculares, contribuyen a facilitar procesos de creación de contenidos multimedia, escenarios de teleformación y entornos colaborativos. Para que una experiencia de este tipo funcione adecuadamente se requiere de un alto grado de coordinación y

García, C., Ribate, M.P., Culleré, L., Fernández, H. y Giner, B. (2020). Elaboración de vídeos educativos sobre operaciones básicas de laboratorio. RIDAS, Revista Iberoamericana de Aprendizaje Servicio, 10, 126-136. DOI10.1344/RIDAS2020.10.11 
predisposición de docentes, así como el compromiso del alumnado.

Se ha alcanzado el objetivo de aprendizaje de los alumnos universitarios con una buena implicación en el proceso de elaboración de los vídeos. Tanto los docentes como los alumnos de Educación Secundaria receptores de los vídeos, y por tanto del servicio, han percibido la experiencia de forma positiva, destacando especialmente la utilidad de estos.

Antes de comenzar a realizar prácticas en un laboratorio conviene conocer el material y cómo se deben realizar las operaciones básicas en el mismo. Actualmente el alumnado de centros de secundaria no suele tener oportunidad de experimentar en un laboratorio. El empleo de vídeos puede ser una herramienta de aprendizaje muy útil para proporcionar una primera toma de contacto virtual consiguiendo así una transición óptima para el alumnado. Los vídeos educativos son medios didácticos que facilitan el descubrimiento de ese tipo de conocimientos además de ser elementos motivadores. Existe la problemática de encontrar vídeos que se ajusten a las necesidades reales que existen en la formación de nuestro alumnado, tanto en la Universidad como en los de Educación Secundaria.

En cuanto a las propuestas de mejora, sería interesante orientar a los alumnos universitarios en la realización de los vídeos proponiéndoles una estructura organizativa (presentación inicial del material, objetivos y posteriormente el cuerpo del vídeo), pudiéndose incrementar la duración de 2 a 3 minutos. Habría también que prestar atención a la calidad de los vídeos, para lo que se podría contar con la colaboración de alumnos o docentes del ámbito de la comunicación audiovisual.

Finalmente, siendo el idioma una limitación reconocida por muchos alumnos que dificulta la atención y comprensión del contenido de los vídeos, deberían incluirse subtítulos para un mejor seguimiento. La elección de la lengua inglesa para la realización de esta actividad amplía la posibilidad de uso de estos vídeos a una población mayor.

Esta experiencia se podría replicar en otras instituciones en las que no se dispone de instalaciones adecuadas para mostrar cómo son los procedimientos desarrollados habitualmente en un laboratorio.

\section{Referencias bibliográficas}

Batlle, R. (2011). ¿De qué hablamos cuando hablamos de aprendizajeservicio? Crítica, (972), 49-54.

Recuperado de: http://www. revistacritica.com/administrator/components/c om avzrevistas/pdfs/b8a385038a9016c af4fb15d0f6c378b8-972-Por-unaeducaci--n-transformadora--mar.abr\%202011.pdf

Bravo Ramos, L. (1996). ¿Qué es el vídeo educativo? Comunicar, (6), 100105. Recuperado de:

https://www.redalyc.org/pdf/158/1580 0620.pdf

Cabero, J. (1995). Propuestas para la utilización del vídeo en los centros. En J. Ballesta (Coord.), Enseñar con los 
medios de comunicación (pp. 89-121). Barcelona, España: Diego Marín (DM) Promociones y Publicaciones Universitarias (PPU).

Cameán, A., Jos, A., Moreno, I., Pichardo, S., Prieto, A. y Repetto, G. (2005). Interés de la elaboración de vídeos didácticos como material de prácticas en la asignatura de "Seguridad Química". Revista de Enseñanza Universitaria, (26), 45-54. Recuperado de: https://idus.us.es/bitstream/handle/11 441/55054/Inter\%c3\%a9s\%20de\%201 a\%20elaboraci\%c3\%b3n\%20N26.pdf?s equence $=1$ \&isAllowed $=y$

García Matamoros, M. (2014). Uso Instruccional del video didáctico. Revista de investigación, 38(81), 4368. Recuperado de:

https://dialnet.unirioja.es/descarga/arti culo/4731936.pdf

Menéndez, M.I., Van der Maelen, J.F. y Pérez, E. (2005). Elaboración de vídeos y de test de autoevaluación como herramienta docente en una asignatura experimental. Revista Latinoamericana de Tecnología Educativa, 4(1), 63-75. Recuperado de: https://relatec.unex.es/article/view/181 $\angle 171$

Pardo, V. (2014). La docencia online: ventajas, inconvenientes y forma de organizarla. Revista Boliviana de Derecho, (18), 622-635. Recuperado de:

https://dialnet.unirioja.es/descarga/arti culo/4766727.pdf

Pérez, E., Rodríguez, J. y García, M. (2014). El uso de mini-vídeos en la práctica docente universitaria. EDMETIC, 4(2), 51-70.

doi:10.21071/edmetic.v4i2.3962

Ruiz-Corbella, M. y García-Gutiérrez, J. (2020). Aprendizaje-Servicio en escenarios digitales de aprendizaje: propuesta innovadora en la educación superior. RIED. Revista Iberoamericana de Educación a Distancia, 23(1), 183198. doi: $10.5944 /$ ried.23.1.24391

Torres, A. (2017). Creación y utilización de vídeo digital y TICs en Física y Química. Eureka Enseñ. Divul. Cien, 6(3), 440-445. Recuperado de: https://revistas.uca.es/index.php/eurek a/article/view/3688/3278

García, C., Ribate, M.P., Culleré, L., Fernández, H. y Giner, B. (2020). Elaboración de vídeos educativos sobre operaciones básicas de laboratorio. RIDAS, Revista Iberoamericana de Aprendizaje Servicio, 10, 126-136. DOI10.1344/RIDAS2020.10.11 